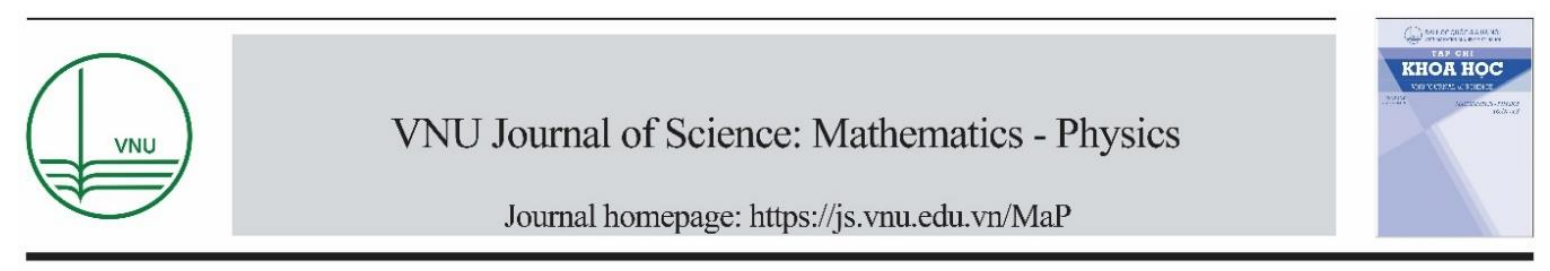

Original Article

\title{
Temperature Gradient: A Simple Method for Single Crystal Growth
}

\author{
Duong Anh Tuan ${ }^{1,2, *}$, Nguyen Thi Thanh Huong ${ }^{3}$, \\ Nguyen Thi Minh $\mathrm{Hai}^{3}$, Pham Anh Tuan ${ }^{3}$, Dinh Thi My Hao ${ }^{4}$, Sunglae $\mathrm{Cho}^{3}$ \\ ${ }^{1}$ Phenikaa Institute for Advanced Study, Phenikaa University, Yen Nghia, Ha Dong, Hanoi, Vietnam \\ ${ }^{2}$ Phenikaa Research and Technology Institute, A\&A Green Phoenix Group, \\ 167 Hoang Ngan, Hanoi, Vietnam \\ ${ }^{3}$ Department of Physics and Energy Harvest-Storage Research Center, University of Ulsan, \\ Ulsan 680-749, Republic of Korea \\ ${ }^{4}$ Department of Physics, Quy Nhon University, 170 An Duong Vuong, Quy Nhon, Vietnam
}

Received 22 December 2018

Revised 21 January 2019; Accepted 25 January 2019

\begin{abstract}
In this article, we provide a simple method for the growth of bulk single crystalline. By the control temperature along a vertical furnace, we can easily fabricate bulk single crystals. This technique is called the temperature gradient method. To create a temperature gradient along the body of the furnace, the density of resistance wire which is coiled along the body of furnace is different. The density increases from the bottom to the top of the furnace. So that, at any time of the growing process, the temperature at the bottom of furnace is the smallest. During could down process, single crystal in the ampoule has been grown up from a seed at the bottom. Using this method, we successfully grew layer structure single crystals such as $\mathrm{SnSe}, \mathrm{SnSe}_{2}, \mathrm{SnS}, \mathrm{GaTe}, \mathrm{InSe}_{2}, \mathrm{GaSe}$. X-ray diffraction and FE-SEM measurements indicated the high quality of single crystals. By changing cooling speed, we can control the number of defects in the single crystals.
\end{abstract}

Keywords: Temperature gradient method, single crystal growth, layer structure materials.

\section{Introduction}

Single crystal usually gives the specific characteristics of materials comparable to polycrystalline and amorphous. It also uses to study the fundamental properties of materials. Base on the fundamental

\footnotetext{
${ }^{*}$ Corresponding author.

E-mail address: tuan.duonganh@phenikaa-uni.edu.vn

https//doi.org/ 10.25073/2588-1124/vnumap.4311
} 
properties of single crystals, we can fully understand the relationship between structure and properties of materials. Furthermore, single crystal has been used in many regions of science and technology. Therefore, many crystal growth techniques were developed from last centuries. Recently, single crystal growth methods are continually improved. The Bridgman technique is the oldest method for growing single crystals. In this technique, the crucible which is contained molten material moves along a gradient temperature of furnace. The crystal is solidified when the bottom of crucible moves to low-temperature region. [1-3] Bridgman technique was developed by Stockbarger by using two separate temperature zones. So, this method is easier for control temperature. [4-6] One of the earliest melting techniques which is used for large single crystal is Kyropoulos method. This method is also grown from melting material in crucible. A seed crystal on the surface of molten material is increased the size by a slowly cooling down of the temperature. [7-9] A similar technique for growing the big size of single crystals is czochralski method. [10-12] But in this method, the seed of crystal is rotated and moved up from surface of molten material. Some other techniques such as Verneuil method and floating zone are also used for single crystal growth. [13-15] All above techniques which are mentioned above are good for growing big size single crystals. However, most of them are complicated. Here, we introduce a simpler technique that is used for growth single crystal. In this method, during growth process, samples do not need moving and we can grow some crystals in a growth process.

\section{Experiment}

This study, we used homemade vertical furnaces which were designed as shown in figure 1 (a). More detail of our furnace will be described in the discussion part. The furnaces are stable working in the temperature range of room temperature to $1000^{\circ} \mathrm{C}$ with a very stable rate of temperature. Materials were loaded into thick wall quartz ampoules. Then, the ampoules were evacuated $\left(<10^{-4}\right.$ Torr $)$ and sealed. Another quartz tube was sealed in order to protect the sample and ampoule when the ampoule breaks during heating due to high vapor pressure of materials and the different thermal expansion coefficient between samples and quartz ampoule. The ampoules and the thermocouple were vertically placed at the bottom of the furnace. In the heating up of growth process, we can select the rate from 10 to $20^{\circ} \mathrm{C} /$ hour which is dependent the material.
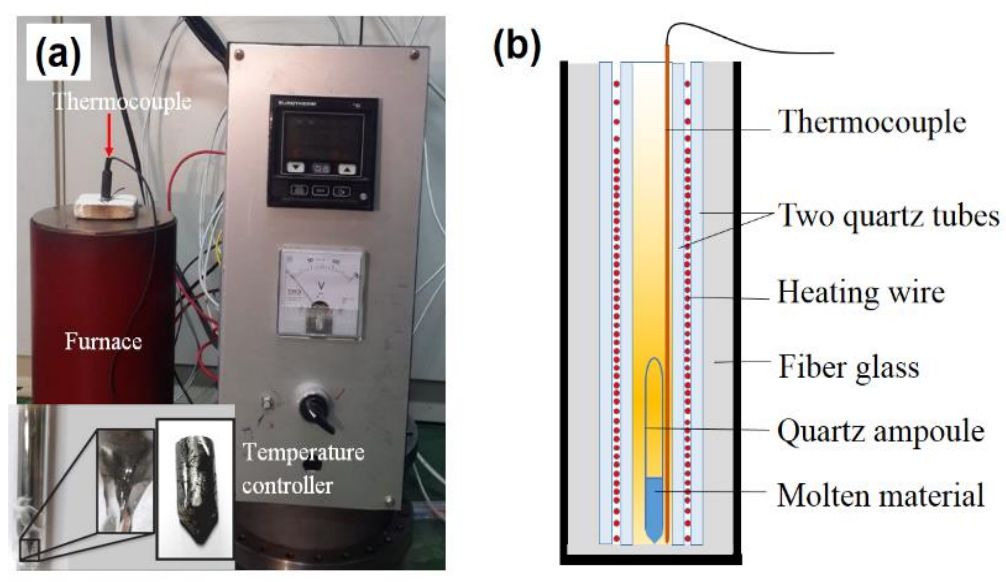

Fig. 1. (a) Photograph of equipment in temperature gradient method. (b) Schematic of the vertical furnace used in the temperature gradient method. 
Maximum temperature is usually higher than melting point of material around $50{ }^{\circ} \mathrm{C}$ and soaked at this temperature around 10 hours before slowly cold down to room temperature. The high purity materials (99.999\%) which were provides by Sigma-Aldrich company should be selected for this growth method. The materials were weighed following the selected stoichiometric ratios before loading into ampoules. The time for a growing process is around 10 to 20 days. X-ray diffraction (XRD) using $\mathrm{Cu}$ $\mathrm{K} \alpha$ radiation (model D/max-RC, Rigaku Co., Tokyo, Japan) and field emission scanning electron microscope (FE-SEM) (mode JSM - 7800F, JEOL Co.) were used to investigate the crystal structure and morphology of grown samples. The composition of grown samples was determined by an energy dispersive spectrometer (EDS) measurement. Some physical properties of samples were investigated.

\section{Results and discussion}

Photography and simulating of the vertical furnace are showed in the Fig. 1. In this method, the temperature was controlled by the multi-step temperature controller and the R-type thermocouple is used as the heating sensor, which is placed at the bottom of the furnace. The gradient temperature in the furnace was created by the different heating wire density. As the result, the temperature at the bottom is a little lower than that the upper region of the furnace. By using this temperature gradient, the solidification process can be initiated from the bottom of the ampoule, which is considered as a crystal seed. From this structure, it is easy to realize that the gradient temperature is strongly depended on the density of heating wire. An important requirement of this growth method is the bottom of quartz ampoule where the seed crystal was formed should be very sharp. Inset of Fig. 1 (a) exhibited the bottom part of quartz ampoule and a grown $\mathrm{SnSe}_{2}$ single crystal. We investigated the temperature attribution in $15 \mathrm{~cm}$ height of furnace by fixing bottom temperature at $800,850,900$, and $950{ }^{\circ} \mathrm{C}$ and another heating sensor was slowly moved up from bottom of furnace to determine the temperature attribution of furnace. The results as shown in Fig. 2 indicated that a temperature gradient was created in the bottom region of furnace. The basic difference between our technique and other previous techniques is in the solidification process. In the previous techniques, the solidification process of the molten material occurs by moving the ampoule or furnace while in our technique, the solidification occurs by the temperature gradient in a single zone of furnace.

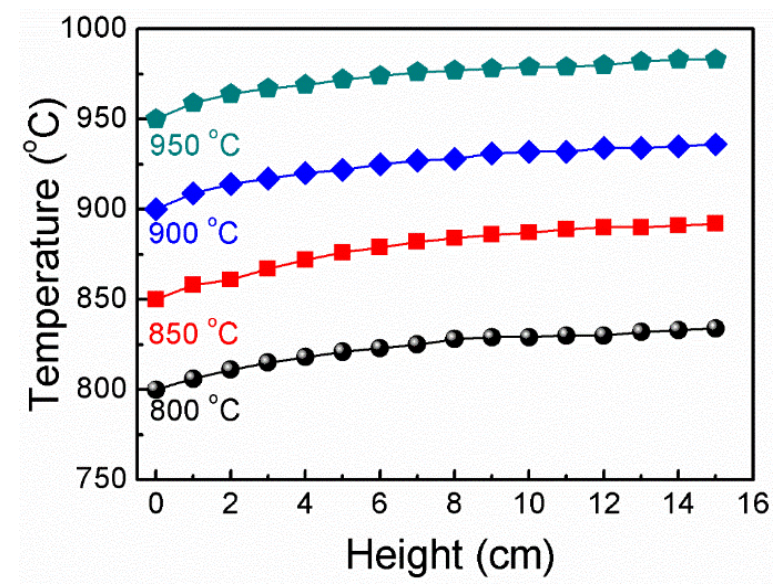

Fig. 2. Temperature profile with different height from the bottom of furnace at the selected fixing of bottom temperatures. 
By using this growth method, we obtained the cylinder single crystals with the size around $1 \mathrm{~cm}$ dimension and 1 to $2 \mathrm{~cm}$ height. Figure 3 shows photographs of some single crystals - (a) SnS, (b) GaSe, (c) $\mathrm{SnSe}_{2}$, and (d) $\mathrm{SnSe}$ which were grown by temperature gradient method. Obtaining single crystals have high uniform. Samples easily cleave with very shiny surface. To evaluate the quality of single crystal grown by this method, the surface of cleaved samples was observed by field emission scanning electron microscope (FE-SEM). The lamellar microstructure with very clean surface and the average thickness around $100 \mathrm{~nm}$ to $1 \mu \mathrm{m}$ of samples were shown in the figure $4(\mathrm{a}-\mathrm{d})$.

Crystal structure of samples grown by this method was characterized by XRD measurement as shown in Figure 5 (a-d). XRD pattern of samples appears only one family peak. Only (h00) diffraction peak family was observed in $\mathrm{SnS}$ and $\mathrm{SnSe}$ samples indicating orthorhombic structure, and the (001) diffraction peak family was appeared in $\mathrm{GaSe}$ and $\mathrm{SnSe}_{2}$ samples indicating hexagonal structure. The XRD results confirmed the quality of single crystals which are grown by temperature gradient method.
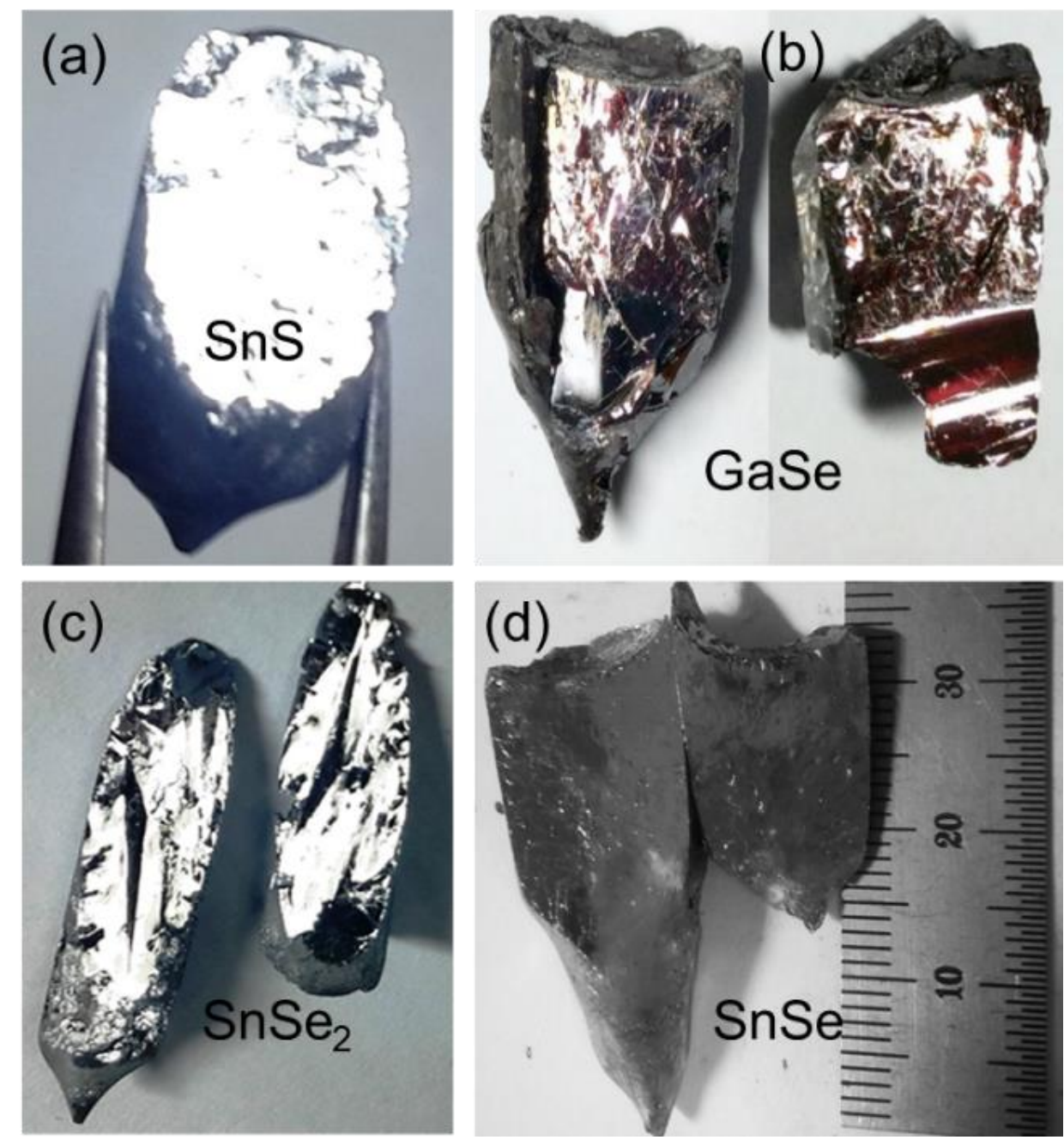

Fig. 3. Photographs of (a) SnS, (b) GaSe, (c) SnSe2, (d) SnSe single crystals grown by temperature gradient method. 

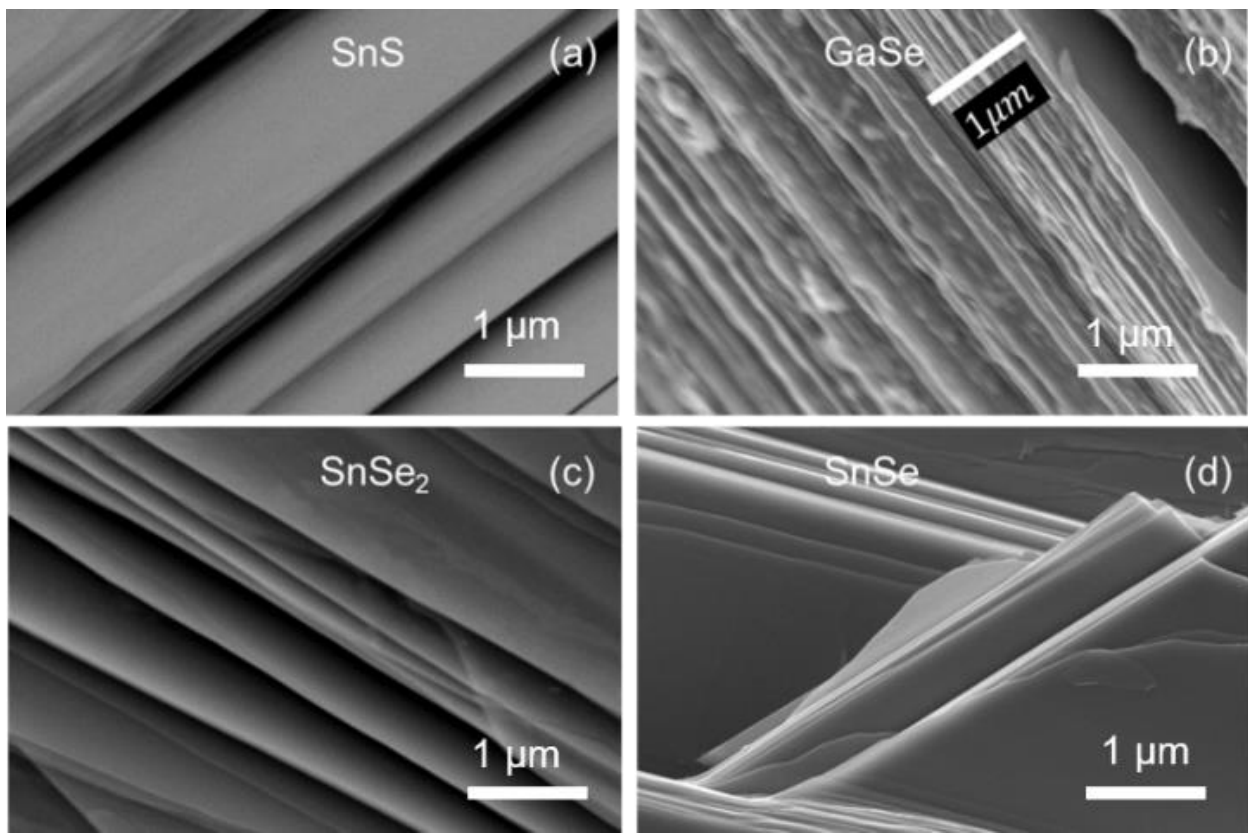

Fig. 4. FE-SEM images of (a) SnS, (b) GaSe, (c) SnSe2, (d) SnSe single crystals grown by temperature gradient method.
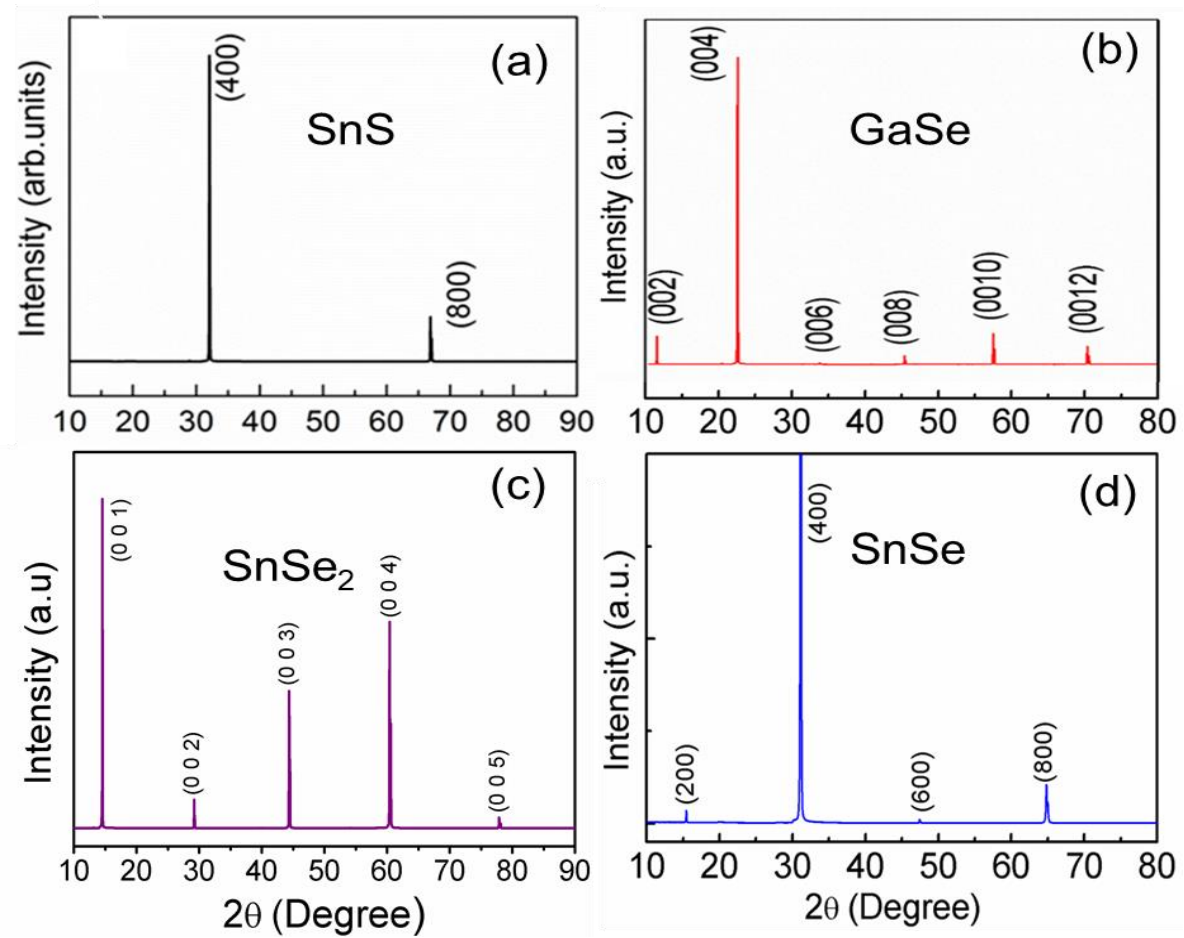

Fig. 5. XRD patterns of (a) $\mathrm{SnS}$, (b) GaSe, (c) $\mathrm{SnSe}$, (d) SnSe single crystals grown by temperature gradient method. 


\section{Conclusions}

Temperature gradient method without the moving of sample is a good method for single crystal fabrication. In one growing process, we can fabricate from one to four crystal with same growth condition. The size of single crystals is around $1 \mathrm{~cm}$ of dimension and 1 to $2 \mathrm{~cm}$ of height. Obtaining crystals have high uniform. Cleaved surface which was observed by FE-SEM is very clean. Only one family of XRD peak in the XRD of sample indicated high quality of single crystal grown by temperature gradient method.

\section{Acknowledgments}

This research is funded by Vietnam National Foundation for Science and Technology Development (NAFOSTED) under grant number 103.02-2016.09

\section{References}

[1] H.J. Scheel, P. Capper, P. Rudolph, Crystal Growth Technology: Semiconductors and Dielectrics, Wiley-VCH Verlag GmbH \& Co. KGaA, Germany, 2010.

[2] C.L. Jones, P. Capper, J.J. Gosney, G. Ard, and I. Kenworthy, Comments on segregation during bridgman growth of CdxHg1-xTe J. Cryst. Growth 64 (1983) 403.

[3] F.R. Szofran and S.L. Lehoczky, A method for interface shape control during Bridgman type crystal growth of HgCdTe alloys, J. Cryst. Growth 70 (1984) 349.

[4] D.M.L. Bartholomew, A. Hellawell, Changes of growth conditions in the vertical Bridgman-Stockbarger method for the solidification of aluminum, J. Cryst. Growth 50 (1980) 453.

[5] W. A. Tiller, K.A. Jacks, J.W. Rutter, B. Chalmers, Ortho-oxy-phenylfluorone, specific reagent either molybdenum or copper, Acta. Met. 1 (1953) 428.

[6] F.E. Pretzel, G.N. Rupert, C.L. Mader, E.K. Siorms, G.V. Gritton, C.C. Rushing, Properties of lithium hydride I. Single crystals, J. Phys. Chem. Solids 16 (1960) 10.

[7] D.F. Bliss, Chap. Evolution and Application of the Kyropoulos Crystal Growth Method, in: 50 Years of Progress in Crystal Growth: A Reprint Collection, Ed. R. Feigelson, Elsevier, (2005), 30-34.

[8] E. Nicklaus, F. Fischer, a modified kyropoulos method for growing BaFCl single crystals, J. Cryst. Growth 12 (1972) 337.

[9] G. Jacob, A novel crystal growth method for GaAs: The liquid encapsulated kyropoulos method, J. Cryst. Growth 58 (1982) 455.

[10] J. Czochralski, a new method for measuring the rate of crystallization of metals, Z. Phys. Chem., 92 (1918) 219.

[11] P.E. Tomaszewski, Jan Czochralski—father of the Czochralski method, J. Cryst. Growth 236 (2002) 1.

[12] N. Tatau, Handbook of Crystal Growth: Fundamentals, Second ed, Amsterdam, Elsevier B.V. the Netherlands (2015)

[13] W. Precht, G.E. Hollox, A floating zone technique for the growth of carbide single crystals, J. Cryst. Growth 3 (1968) 818.

[14] J.D. Verhoeven, E. D. Gibson, M. A. Noack, and R. J. Conzemius, An arc floating zone technique for preparing single crystal lanthanum hexaboride, J. Cryst. Growth 36 (1976) 115.

[15] R.C.Pastor, A.C. Pastor, Crystal growth above $2200^{\circ} \mathrm{C}$ by the Verneuil method, Mater. Research Bulletin 1 (1966) 275. 